\section{Tendências nas condições de saúde e uso de serviços de saúde entre idosos brasileiros: um estudo baseado na Pesquisa Nacional por Amostra de Domicílios (1998, 2003)}

\author{
Trends in health conditions and use of health \\ services by the Brazilian elderly: a study based on \\ the National Household Sample Survey
}

(1998, 2003)

\begin{abstract}
This study examined trends in health conditions and use of health services by the Brazilian elderly, based on health data from the National Household Sample Surveys (PNAD) conducted in 1998 and 2003. 28,943 and 35,042 individuals aged $\geq 60$ years were included in the study, respectively. The results showed an improve ment in health conditions in the study population during this period, as measured by selfrated health, having remained bedridden in the previous two weeks, ability to perform selected activities of daily living, number of chronic conditions, and self-reported arthritis. There was also an increase in the number of doctor and dentist visits from 1998 to 2003. The trends were consistent for both men and women. The results emphasize the importance of the PNAD health supplement as a source of information for the surveillance or monitoring of health and health-related conditions in the Brazilian elderly population.
\end{abstract}

Aged; Health Services; Health Services Research; Health Status
Maria Fernanda Lima-Costa 1

Antônio I. de Loyola Filho ${ }^{1}$

Divane L. Matos 1

Os problemas de saúde e o uso de serviços de saúde aumentam com a idade, sobretudo nas últimas décadas de vida. Dessa forma, em sociedades mais envelhecidas e/ou em rápido processo de envelhecimento, como é o caso do Brasil, torna-se necessário conhecer as tendências em saúde da população idosa. O monitoramento e/ou a vigilância ${ }^{1}$ das condições de saúde e seus determinantes têm o objetivo de detectar mudanças ao longo do tempo para subsidiar políticas sociais.

De uma maneira geral, os indicadores utilizados nos sistemas de informações para o monitoramento das condições de saúde e seus determinantes entre idosos são baseados em informações produzidas rotineiramente - entre as quais a mortalidade é o exemplo mais visível - e em informações obtidas por meio de inquéritos de saúde 2,3. No Canadá, o sistema de informações busca responder a cinco perguntas: (1) quão saudáveis são os idosos? (2) como o sistema de saúde está servindo aos idosos? (3) qual a situação econômica do idoso? (4) quais as condições de vida dos idosos? e (5) como os idosos estão participando da sociedade? Entre as condições de saúde, são consideradas a expectativa de vida saudável e a esperança de vida aos 65 anos de idade, assim como percepção da saúde, presença de doenças crônicas, limitações nas atividades da vida diária, alguns estilos de vida (exercício físico 
e tabagismo), hospitalizações devido a quedas e acidentes, suicídio e senso de coerência. Para avaliar como o sistema de saúde está servindo ao idoso, são considerados consultas médicas, hospitalizações, consumo de medicamentos, cuidado domiciliar, institucionalização, acesso e gastos com a saúde 2. Nos Estados Unidos, o sistema de informações contempla dados sócio-demográficos e condições de vida, mas é mais centrado nas condições de saúde, incluindo indicadores de morbidade e de incapacidade, mortalidade e uso e gastos com serviços de saúde 3 .

No Brasil, não existem sistemas de informações que atendam às particularidades da população idosa. Os dados disponíveis sobre as condições de saúde e uso de serviços de saúde dessa população são aqueles baseados na mortalidade, nas hospitalizações no âmbito do Sistema Único de Saúde (SUS), nas doenças de notificação obrigatória e em outras informações produzidas pelos programas e serviços de saúde ${ }^{4}$. As informações obtidas por meio de inquéritos de saúde ainda não são consideradas como parte desses sistemas.

A Pesquisa Nacional por Amostra de Domicílios (PNAD), realizada pelo Instituto Brasileiro de Geografia e Estatística (IBGE), é um inquérito de base populacional, com abrangência nacional. Realizada anualmente, a pesquisa produz informações sobre características demográficas, habitação, educação, trabalho e rendimentos da população brasileira. Em 1998 e 2003, a pesquisa incluiu um suplemento 5,6 sobre saúde.

Baseado no suplemento da PNAD de 1998, acima mencionado, foi realizado um trabalho para examinar as condições de saúde e uso de serviços de saúde entre idosos brasileiros. Os resultados mostraram que as condições de saúde dessa população eram muito semelhantes às de outras populações idosas. Da mesma forma, o uso de serviços de saúde, definido por consultas médicas e hospitalizações, estava dentro das variações internacionais 7. O presente trabalho é uma continuação dessa pesquisa. O estudo, baseado na PNAD, tem por objetivo verificar as tendências - entre 1998 e 2003 - das condições de saúde e do uso de serviços de saúde pela população idosa brasileira.

\section{Metodologia}

\section{População estudada}

A PNAD é baseada em uma amostra probabilística de domicílios obtida em três estágios de seleção. No primeiro estágio é feita a seleção dos municípios, que são classificados em auto-re- presentativos, com probabilidade 1 de pertencer à amostra, e em não auto-representativos, com probabilidade de pertencer à amostra proporcional à população residente. No segundo estágio, são selecionados os setores censitários, cuja probabilidade de inclusão é proporcional ao número de domicílios existentes no setor. No terceiro estágio, os domicílios são amostrados em cada setor censitário, sendo investigadas, por meio de entrevista, as informações relativas a todos os residentes no domicílio. A PNAD 1998 foi conduzida entre os dias 20 e 26 de setembro de 1998 5. A PNAD 2003 foi conduzida entre os dias 21 e 27 de setembro de 2003 6. Para o presente trabalho foram selecionados todos os participantes da PNAD 1998 e da PNAD 2003 com sessenta ou mais anos de idade 5,6 .

\section{Indicadores utilizados}

Foram considerados indicadores da condição de saúde o uso de serviços de saúde e cobertura por plano de saúde. Sexo e idade foram as características demográficas consideradas.

As condições de saúde auto-referidas foram examinadas, utilizando-se indicadores gerais (percepção da saúde, interrupção das atividades habituais devido a problemas de saúde nas últimas duas semanas e ter estado acamado nesse período) e específicos dessas condições (número de doenças crônicas e doenças autoreferidas selecionadas), além da capacidade funcional (grau de dificuldade para realizar pelo menos uma entre três atividades da vida diária: alimentar-se, tomar banho e/ou ir ao banheiro). As doenças crônicas consideradas neste trabalho foram hipertensão arterial, artrite/reumatismo e diabetes. Para o cálculo do número de doenças crônicas foram consideradas todas aquelas pesquisadas pela PNAD, incluindo hipertensão, artrite/reumatismo, doença do coração, diabetes, asma/bronquite, doença renal crônica, câncer, cirrose, depressão e doença da coluna/dor nas costas 5,6 .

Entre os indicadores de usos de serviços de saúde foram considerados: número de consultas médicas nos últimos 12 meses e número de hospitalizações nos últimos 12 meses. Filiação a plano de saúde foi considerada como qualquer resposta afirmativa a ter plano de saúde médico ou odontológico, particular, de empresa ou órgão público 5,6.

\section{Análise dos dados}

A análise foi realizada, incorporando-se os pesos do indivíduo (inverso da fração amostral) e a correção para efeito do delineamento do estudo, 
utilizando-se procedimentos para inquéritos populacionais do programa Stata versão 7.0 (Stata Corp., College Station, Estados Unidos). Foram calculadas as taxas de prevalência e intervalos de confiança no nível de 95\%, considerando-se a faixa etária de sessenta ou mais anos de idade, para homens e mulheres, assim como para ambos os sexos. Razões de prevalência ajustadas por idade (variável contínua) e sexo foram utilizados para comparar as taxas de prevalência observadas em 2003, em relação a 1998. As estimativas das razões de prevalência ajustadas foram feitas por meio da regressão de Poisson ${ }^{8}$. De forma complementar, a distribuição das características investigadas foi estratificada por sexo e faixa etária (60-69, 70-79 e 80 e + anos). Todas as análises foram realizadas utilizando-se o programa Stata versão 7.0.

\section{Resultados}

Este trabalho incluiu 28.943 participantes da PNAD 1998 e 35.042 participantes da PNAD 2003 com sessenta ou mais anos de idade. A média de idade dos participantes foi igual a 69,5 anos (variação = 60,0-113,0) em 1998 e 69,8 anos (variação $=60,0-106,0)$ em 2003. A porcentagem de mulheres nos anos correspondentes foi igual a 55,5 e 56,0, respectivamente.

Na Tabela 1 está apresentada a distribuição percentual de indicadores da condição de saúde e uso de serviços entre idosos de ambos os sexos, segundo o ano. Entre 1998 e 2003, foram observadas reduções significativas $(p<0,05)$ e independentes da idade e do sexo para: percepção da saúde como ruim ou muito ruim, ter estado acamado em período recente, pequena e grande dificuldade para realizar atividades da vida diária, artrite/reumatismo auto-referido e número de doenças crônicas auto-referidas (2, 3 ou mais). Aumentos significantes e independentes foram observados para hipertensão e diabetes auto-referidos, assim como para o número de consultas médicas (1, 2, 3 ou mais). Não foram observadas mudanças significativas na prevalência da interrupção das atividades habituais devido a problemas de saúde nas duas últimas semanas, no número de hospitalizações no último ano e na prevalência da cobertura por plano de saúde.

Os resultados acima mencionados foram semelhantes em homens (Tabela 2) e mulheres (Tabela 3), com pequenas exceções. As exceções foram as seguintes: (1) entre os homens, não foi observada redução significante na prevalência da percepção da saúde como razoável, ao passo que entre as mulheres essa redução foi significante; (2) a redução do número de doenças crônicas auto-referidas, entre os homens, foi observada nos três estratos analisados, enquanto, entre as mulheres, essa redução foi observada somente para 2 e 3 ou mais doenças.

Nas Tabelas 4 e 5 estão apresentadas as distribuições percentuais dos indicadores das condições de saúde e de uso de serviços de saúde entre homens e mulheres, respectivamente, segundo a faixa etária. Em ambos os sexos, tanto em 1998 quanto em 2003, verificou-se aumento gradual com a idade da pior percepção da saúde (ruim/muito ruim), da interrupção recente das atividades habituais devido a problemas de saúde, de ter estado acamado, da artrite/reumatismo, do grau de dificuldade para realizar atividades da vida diária (pequeno, grande e total incapacidade), do maior tempo decorrido após a última visita ao dentista (três ou mais anos e nunca ter ido) e do número de hospitalizações no último ano (uma e duas ou mais). O número de consultas médicas no último ano (três ou mais consultas) aumentou com a idade entre os idosos, mas não entre as idosas. As prevalências dos demais indicadores considerados neste trabalho não se modificaram com a idade ou não apresentaram tendências claras de aumento ou diminuição.

\section{Discussão}

Os resultados do presente trabalho apontam para melhora nas condições de saúde auto-referidas dos idosos brasileiros, no período compreendido entre 1998 e 2003, considerando-se indicadores gerais (percepção da saúde e ter estado recentemente acamado, assim como grau de dificuldade para realizar atividades da vida diária) e específicos das condições de saúde (número de doenças crônicas e relato de artrite/reumatismo). Os resultados também apontam para aumento do número de consultas médicas. É importante salientar que as tendências acima mencionadas foram observadas em ambos os sexos.

A percepção da saúde, ou auto-avaliação da saúde, é um dos indicadores mais utilizados em pesquisas gerontológicas. O seu uso é justificado porque a pior percepção da saúde é um preditor robusto e consistente da mortalidade 9,10. Além disso, o risco da mortalidade associado à pior percepção da saúde é maior do que o risco associado a medidas objetivas da condição de saúde 11,12. A percepção da saúde tem sido também utilizada para examinar tendências nas condições de saúde da população idosa, em países como o Canadá 2 e os Estados Unidos 3. No Brasil, a PNAD 5,6 é a principal fonte de informações sobre a percepção da saúde da população idosa. A 
Distribuição percentual de indicadores da condição de saúde e uso de serviços de saúde entre homens e mulheres com 60 ou mais anos de idade. Pesquisa Nacional por Amostra de Domicílios (PNAD), 1998, 2003 5,6.

\begin{tabular}{|c|c|c|c|c|c|c|}
\hline \multirow[t]{2}{*}{ Indicadores } & \multicolumn{2}{|c|}{ PNAD 1998} & \multicolumn{2}{|c|}{ PNAD 2003} & \multirow[t]{2}{*}{ OR * } & \multirow[t]{2}{*}{ IC95\% } \\
\hline & $\%$ & IC95\% & $\%$ & IC95\% & & \\
\hline \multicolumn{7}{|l|}{ Percepção da saúde } \\
\hline Muito boa/Boa & 39,3 & $37,1-41,6$ & 43,6 & $40,6-46,7$ & 1,00 & \\
\hline Regular & 44,2 & $43,0-45,4$ & 42,9 & $41,2-44,5$ & 0,87 & $0,76-1,00$ \\
\hline Ruim/Muito ruim & 16,6 & $15,2-18,0$ & 13,6 & $12,1-15,1$ & 0,73 & $0,58-0,91$ \\
\hline $\begin{array}{l}\text { Interrupção das atividades habituais por } \\
\text { problema de saúde nas duas últimas semanas ** }\end{array}$ & 13,9 & $13,3-14,5$ & 13,2 & $12,2-14,1$ & 0,93 & $0,84-1,03$ \\
\hline Ter estado acamado nas duas últimas semanas ** & 9,5 & $9,0-9,9$ & 8,4 & $7,9-8,9$ & 0,87 & $0,80-0,94$ \\
\hline \multicolumn{7}{|l|}{ Grau de dificuldade para realizar } \\
\hline \multicolumn{7}{|l|}{ atividades da vida diária $* \star \star$} \\
\hline Nenhum & 84,6 & $83,4-85,7$ & 86,6 & $85,8-87,3$ & 1,00 & \\
\hline Pequeno & 8,9 & $8,1-9,8$ & 7,1 & $6,5-7,7$ & 0,76 & $0,66-0,87$ \\
\hline Grande & 4,5 & $4,2-4,9$ & 4,0 & $3,7-4,3$ & 0,84 & $0,74-0,95$ \\
\hline Não consegue & 2,0 & $1,8-2,3$ & 2,4 & $2,1-2,7$ & 1,09 & $0,90-1,31$ \\
\hline \multicolumn{7}{|l|}{ Doenças crônicas auto-referidas } \\
\hline Hipertensão & 43,9 & $43,0-44,9$ & 48,8 & $47,6-50,0$ & 1,22 & $1,14-1,29$ \\
\hline Artrite/Reumatismo & 37,5 & $35,4-40,0$ & 27,3 & $25,4-29,2$ & 0,61 & $0,53-0,70$ \\
\hline Diabetes & 10,3 & $9,6-11,0$ & 13,0 & $12,2-13,9$ & 1,30 & $1,17-1,45$ \\
\hline \multicolumn{7}{|l|}{ Número de doenças crônicas auto-referidas } \\
\hline Nenhuma & 21,6 & $20,4-22,9$ & 24,9 & $23,8-26,1$ & 1,00 & \\
\hline 1 & 24,9 & $24,2-25,5$ & 27,3 & $26,6-28,1$ & 0,95 & $0,87-1,03$ \\
\hline 2 & 23,3 & $22,7-23,9$ & 22,2 & $21,7-22,8$ & 0,82 & $0,74-0,90$ \\
\hline 3 ou + & 30,3 & $29,0-31,5$ & 25,6 & $24,4-26,8$ & 0,72 & $0,63-0,82$ \\
\hline \multicolumn{7}{|l|}{ Número de consultas a um médico nos } \\
\hline \multicolumn{7}{|l|}{ últimos 12 meses } \\
\hline Nenhuma & 27,8 & $26,5-29,2$ & 22,0 & $20,3-23,7$ & 1,00 & \\
\hline 1 & 13,9 & $13,0-14,8$ & 12,7 & $11,9-13,7$ & 1,17 & $1,07-1,27$ \\
\hline 2 & 14,0 & $13,5-14,5$ & 14,3 & $13,8-14,7$ & 1,29 & $1,18-1,42$ \\
\hline 3 ou + & 44,3 & $42,2-46,4$ & 51,0 & $48,5-53,5$ & 1,47 & $1,26-1,71$ \\
\hline \multicolumn{7}{|l|}{ Tempo decorrido da última visita ao dentista (anos) } \\
\hline$<1$ & 13,2 & $11,4-15,2$ & 17,4 & $15,2-19,8$ & 1,00 & \\
\hline $1-2$ & 9,5 & $8,4-10,8$ & 11,0 & $9,7-12,4$ & 0,88 & $0,80-0,96$ \\
\hline 3 ou + & 71,1 & $68,6-73,3$ & 65,8 & $62,9-68,5$ & 0,69 & $0,54-0,88$ \\
\hline Nunca & 6,3 & $5,3-7,4$ & 5,9 & $4,6-7,5$ & 0,69 & $0,44-1,08$ \\
\hline \multicolumn{7}{|l|}{ Número de hospitalizações nos últimos 12 meses } \\
\hline Nenhuma & 86,4 & $85,6-87,1$ & 87,3 & $86,2-88,3$ & 1,00 & \\
\hline 1 & 9,3 & $8,8-9,8$ & 8,7 & $8,1-9,3$ & 0,92 & $0,83-1,02$ \\
\hline $2 \mathrm{ou}+$ & 4,3 & $4,0-4,7$ & 4,0 & $3,5-4,6$ & 0,91 & $0,76-1,09$ \\
\hline Cobertura por plano de saúde ** & 26,9 & $22,1-32,2$ & 29,4 & $25,0-34,3$ & 1,14 & $0,81-1,60$ \\
\hline
\end{tabular}

* Odds ratio (OR) ajustado por idade e sexo;

** Percentual de respostas afirmativas;

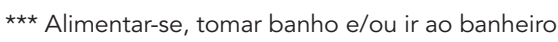


Distribuição percentual de indicadores da condição de saúde e uso de serviços de saúde entre homens com 60 ou mais anos de idade. Pesquisa Nacional por Amostra de Domicílios (PNAD), 1998, 2003 5,6.

\begin{tabular}{|c|c|c|c|c|c|c|}
\hline \multirow[t]{2}{*}{ Indicadores } & \multicolumn{2}{|c|}{ PNAD 1998} & \multicolumn{2}{|c|}{ PNAD 2003} & \multirow[t]{2}{*}{$\mathrm{OR}$ * } & \multirow[t]{2}{*}{ IC95\% } \\
\hline & $\%$ & IC95\% & $\%$ & $1 \mathrm{C} 95 \%$ & & \\
\hline \multicolumn{7}{|l|}{ Percepção da saúde } \\
\hline Muito boa/Boa & 41,6 & $39,6-43,6$ & 45,1 & $42,1-48,2$ & 1,00 & \\
\hline Regular & 42,3 & $41,1-43,5$ & 41,7 & $39,9-43,6$ & 0,90 & $0,79-1,03$ \\
\hline Ruim/Muito ruim & 16,1 & $14,7-17,6$ & 13,2 & $11,7-14,8$ & 0,74 & $0,60-0,92$ \\
\hline Interrupção das atividades habituais por & 12,8 & $12,1-13,5$ & 11,9 & $10,9-13,1$ & 0,92 & $0,82-1,03$ \\
\hline \multicolumn{7}{|l|}{ problema de saúde nas duas últimas semanas ** } \\
\hline Ter estado acamado nas duas últimas semanas ** & 8,3 & $7,8-8,9$ & 7,3 & $6,8-7,8$ & 0,85 & $0,77-0,95$ \\
\hline \multicolumn{7}{|l|}{ Grau de dificuldade para realizar } \\
\hline \multicolumn{7}{|l|}{ atividades da vida diária *** } \\
\hline Nenhum & 86,7 & $85,4-87,8$ & 88,5 & $87,7-89,3$ & 1,00 & \\
\hline Pequeno & 7,6 & $6,9-8,5$ & 5,9 & $5,4-6,5$ & 0,74 & $0,63-0,86$ \\
\hline Grande & 3,9 & $3,5-4,4$ & 3,6 & $3,2-3,9$ & 0,85 & $0,73-1,00$ \\
\hline Não consegue & 1,8 & $1,5-2,1$ & 2,0 & $1,7-2,4$ & 1,07 & $0,83-1,37$ \\
\hline \multicolumn{7}{|l|}{ Doenças crônicas auto-referidas } \\
\hline Hipertensão & 36,6 & $35,6-37,7$ & 40,4 & $39,0-41,9$ & 1,17 & $1,10-1,26$ \\
\hline Artrite/Reumatismo & 30,0 & $27,7-32,3$ & 20,1 & $18,3-22,0$ & 0,58 & $0,50-0,68$ \\
\hline Diabetes & 8,7 & $8,0-9,5$ & 11,9 & $10,8-13,0$ & 1,40 & $1,22-1,62$ \\
\hline \multicolumn{7}{|l|}{ Número de doenças crônicas auto-referidas } \\
\hline Nenhuma & 26,5 & $25,0-28,0$ & 31,0 & $29,6-32,5$ & 1,00 & \\
\hline 1 & 28,1 & $27,2-29,0$ & 29,1 & $28,3-30,0$ & 0,88 & $0,81-0,96$ \\
\hline 2 & 21,9 & $21,0-22,9$ & 20,9 & $20,1-21,7$ & 0,81 & $0,72-0,91$ \\
\hline 3 ou + & 23,5 & $22,2-24,8$ & 19,0 & $17,9-20,1$ & 0,68 & $0,59-0,78$ \\
\hline \multicolumn{7}{|l|}{ Número de consultas a um médico nos } \\
\hline \multicolumn{7}{|l|}{ últimos 12 meses } \\
\hline Nenhuma & 34,7 & $33,2-36,1$ & 28,8 & $26,7-31,1$ & 1,00 & \\
\hline 1 & 15,0 & $14,1-16,0$ & 14,4 & $13,5-15,3$ & 1,15 & $1,02-1,28$ \\
\hline 2 & 13,6 & $12,9-14,2$ & 14,2 & $13,5-14,9$ & 1,26 & $1,12-1,41$ \\
\hline $3 \mathrm{ou}+$ & 36,8 & $34,9-38,7$ & 42,6 & $40,1-45,1$ & 1,39 & $1,18-1,63$ \\
\hline \multicolumn{7}{|l|}{ Tempo decorrido da última visita ao dentista (anos) } \\
\hline$<1$ & 14,0 & $12,3-15,8$ & 18,3 & $15,9-20,9$ & 1,00 & \\
\hline $1-2$ & 9,9 & $8,6-11,4$ & 11,3 & $10,0-12,7$ & 0,87 & $0,78-0,97$ \\
\hline $3 \mathrm{ou}+$ & 68,6 & $66,2-70,8$ & 63,6 & $60,7-66,4$ & 0,70 & $0,55-0,88$ \\
\hline Nunca & 7,5 & $6,4-8,8$ & 6,9 & $5,3-8,9$ & 0,68 & $0,44-1,06$ \\
\hline \multicolumn{7}{|l|}{ Número de hospitalizações nos últimos 12 meses } \\
\hline Nenhuma & 86,1 & $85,2-86,9$ & 86,9 & $85,8-87,9$ & 1,00 & \\
\hline 1 & 9,6 & $9,0-10,2$ & 9,0 & $8,4-9,7$ & 0,93 & $0,84-1,03$ \\
\hline $2 \mathrm{ou}+$ & 4,4 & $4,0-4,8$ & 4,1 & $3,5-4,7$ & 0,92 & $0,76-1,10$ \\
\hline Cobertura por plano de saúde ** & 24,8 & $20,3-29,9$ & 27,2 & $22,7-32,3$ & 1,14 & $0,80-1,62$ \\
\hline
\end{tabular}

* Odds ratio (OR) ajustado por idade e sexo;

** Percentual de respostas afirmativas;

*** Alimentar-se, tomar banho e/ou ir ao banheiro. 
Distribuição percentual de indicadores da condição de saúde e uso de serviços de saúde entre mulheres com 60 ou mais anos de idade. Pesquisa Nacional por Amostra de Domicílios (PNAD), 1998, 2003 5,6.

\begin{tabular}{|c|c|c|c|c|c|c|}
\hline \multirow[t]{2}{*}{ Indicadores } & \multicolumn{2}{|c|}{ PNAD 1998} & \multicolumn{2}{|c|}{ PNAD 2003} & \multirow[t]{2}{*}{$\mathrm{OR}$ * } & \multirow[t]{2}{*}{ IC95\% } \\
\hline & $\%$ & IC95\% & $\%$ & IC95\% & & \\
\hline \multicolumn{7}{|l|}{ Percepção da saúde } \\
\hline Muito boa/Boa & 37,5 & $34,8-40,2$ & 42,4 & $39,3-45,6$ & 1,00 & \\
\hline Regular & 45,6 & $44,1-47,2$ & 43,7 & $42,1-45,4$ & 0,84 & $0,73-0,98$ \\
\hline Ruim/Muito ruim & 16,9 & $15,5-18,5$ & 13,8 & $12,4-15,5$ & 0,71 & $0,56-0,91$ \\
\hline \multicolumn{7}{|l|}{ problema de saúde nas duas últimas semanas ** } \\
\hline Ter estado acamado nas duas últimas semanas ** & 10,4 & $9,8-11,0$ & 9,3 & $8,7-10,0$ & 0,88 & $0,79-0,97$ \\
\hline \multicolumn{7}{|l|}{ Grau de dificuldade para realizar } \\
\hline \multicolumn{7}{|l|}{ atividades da vida diária *** } \\
\hline Nenhum & 82,9 & $81,7-84,1$ & 85,0 & $84,0-86,0$ & 1,00 & \\
\hline Pequeno & 9,9 & $9,0-10,9$ & 8,0 & $7,3-8,7$ & 0,77 & $0,66-0,89$ \\
\hline Grande & 5,0 & $4,5-5,5$ & 4,4 & $4,0-4,8$ & 0,83 & $0,72-0,95$ \\
\hline Não consegue & 2,2 & $1,9-2,5$ & 2,6 & $2,3-3,0$ & 1,11 & $0,90-1,36$ \\
\hline \multicolumn{7}{|l|}{ Doenças crônicas auto-referidas } \\
\hline Hipertensão & 49,7 & $48,4-51,1$ & 55,3 & $54,1-56,6$ & 1,25 & $1,16-1,35$ \\
\hline Artrite/Reumatismo & 43,6 & $41,4-45,8$ & 33,0 & $30,7-35,3$ & 0,63 & $0,55-0,72$ \\
\hline Diabetes & 11,5 & $10,8-12,3$ & 13,9 & $13,1-14,7$ & 1,24 & $1,12-1,37$ \\
\hline \multicolumn{7}{|l|}{ Número de doenças crônicas auto-referidas } \\
\hline Nenhuma & 17,7 & $16,4-19,0$ & 20,1 & $19,1-21,1$ & 1,00 & \\
\hline 1 & 22,3 & $21,6-23,0$ & 25,9 & $25,0-26,8$ & 1,02 & $0,93-1,13$ \\
\hline 2 & 24,4 & $23,7-25,0$ & 23,3 & $22,7-23,9$ & 0,84 & $0,75-0,93$ \\
\hline 3 ou + & 35,7 & $34,2-37,2$ & 30,8 & $29,3-32,3$ & 0,76 & $0,65-0,87$ \\
\hline \multicolumn{7}{|l|}{ Número de consultas a um médico } \\
\hline \multicolumn{7}{|l|}{ nos últimos 12 meses } \\
\hline Nenhuma & 22,4 & $21,1-23,8$ & 16,6 & $15,3-17,9$ & 1,00 & \\
\hline 1 & 13,0 & $12,0-14,0$ & 11,5 & $10,4-12,6$ & 1,20 & $1,08-1,32$ \\
\hline 2 & 14,4 & $13,7-15,1$ & 14,3 & $13,6-15,0$ & 1,34 & $1,21-1,49$ \\
\hline 3 ou + & 50,3 & $48,0-52,6$ & 57,7 & $55,2-60,1$ & 1,55 & $1,33-1,80$ \\
\hline \multicolumn{7}{|l|}{ Tempo decorrido da última visita } \\
\hline \multicolumn{7}{|l|}{ ao dentista (anos) } \\
\hline$<1$ & 12,5 & $10,6-14,8$ & 16,7 & $14,5-19,2$ & 1,00 & \\
\hline $1-2$ & 9,1 & $8,1-10,4$ & 10,7 & $9,4-12,3$ & 0,88 & $0,78-0,99$ \\
\hline $3 \mathrm{ou}+$ & 73,0 & $70,4-75,5$ & 67,5 & $64,4-70,5$ & 0,68 & $0,52-0,90$ \\
\hline Nunca & 5,3 & $4,4-6,4$ & 5,1 & $3,9-6,5$ & 0,69 & $0,43-1,12$ \\
\hline \multicolumn{7}{|l|}{ Número de hospitalizações nos } \\
\hline \multicolumn{7}{|l|}{ últimos 12 meses } \\
\hline Nenhuma & 86,6 & $85,7-87,5$ & 87,6 & $86,2-88,9$ & 1,00 & \\
\hline 1 & 9,1 & $8,5-9,7$ & 8,4 & $7,6-9,3$ & 0,92 & $0,80-1,05$ \\
\hline $2 \mathrm{ou}+$ & 4,3 & $3,9-4,8$ & 4,0 & $3,4-4,7$ & 0,90 & $0,73-1,11$ \\
\hline Cobertura por plano de saúde ** & 28,6 & $23,6-34,1$ & 31,2 & $26,6-36,1$ & 1,13 & $0,81-1,59$ \\
\hline
\end{tabular}

* Odds ratio (OR) ajustado por idade e sexo;

** Percentual de respostas afirmativas;

*** Alimentar-se, tomar banho e/ou ir ao banheiro. 
Distribuição percentual de indicadores da condição de saúde e uso de serviços de saúde entre homens, segundo a faixa etária. Pesquisa Nacional por Amostra de Domicílios (PNAD), 1998, 2003 5,6.

\begin{tabular}{|c|c|c|c|c|c|c|}
\hline \multirow[t]{3}{*}{ Indicadores } & \multicolumn{3}{|c|}{ PNAD 1998} & \multirow{2}{*}{\multicolumn{3}{|c|}{$\begin{array}{c}\text { PNAD } 2003 \\
\text { Faixa etária (anos) }\end{array}$}} \\
\hline & \multicolumn{3}{|c|}{ Faixa etária (anos) } & & & \\
\hline & $60-69$ & 70-79 & $80 \mathrm{e}+$ & $60-69$ & 70-79 & $80 \mathrm{e}+$ \\
\hline \multicolumn{7}{|l|}{ Percepção da saúde } \\
\hline Muito boa/Boa & 46,1 & 36,4 & 31,1 & 49,9 & 40,4 & 33,5 \\
\hline Regular & 40,7 & 44,5 & 45,2 & 39,5 & 44,8 & 44,7 \\
\hline Ruim/Muito ruim & 13,2 & 19,1 & 23,8 & 10,6 & 14,8 & 21,8 \\
\hline Interrupção das atividades habituais por & 11,1 & 13,7 & 19,3 & 10,0 & 13,4 & 17,9 \\
\hline \multicolumn{7}{|l|}{ problemas de saúde nas duas últimas semanas * } \\
\hline Ter estado acamado nas duas últimas semanas ** & 6,9 & 9,2 & 14,2 & 5,8 & 8,1 & 12,7 \\
\hline \multicolumn{7}{|l|}{ Grau de dificuldade para realizar atividades } \\
\hline \multicolumn{7}{|l|}{ da vida diária ** } \\
\hline Nenhum & 90,6 & 85,3 & 68,3 & 93,3 & 86,1 & 70,3 \\
\hline Pequeno & 5,9 & 8,3 & 15,5 & 3,8 & 7,2 & 13,4 \\
\hline Grande & 2,8 & 4,1 & 10,4 & 2,0 & 4,1 & 10,0 \\
\hline Não consegue & 0,8 & 2,3 & 6,0 & 0,9 & 2,6 & 6,3 \\
\hline \multicolumn{7}{|l|}{ Doenças crônicas auto-referidas } \\
\hline Hipertensão & 35,8 & 38,5 & 36,0 & 37,6 & 44,9 & 42,6 \\
\hline Artrite/Reumatismo & 27,0 & 33,0 & 38,2 & 16,8 & 23,1 & 28,5 \\
\hline Diabetes & 8,5 & 9,4 & 8,1 & 10,7 & 13,5 & 13,2 \\
\hline \multicolumn{7}{|l|}{ Número de doenças crônicas auto-referidas } \\
\hline Nenhuma & 28,1 & 24,3 & 23,6 & 34,5 & 26,6 & 26,1 \\
\hline 1 & 28,5 & 27,8 & 27,1 & 30,0 & 28,6 & 26,2 \\
\hline 2 & 21,9 & 21,7 & 22,9 & 19,3 & 22,7 & 24,2 \\
\hline 3 ou + & 21,6 & 26,3 & 26,4 & 16,3 & 22,2 & 23,6 \\
\hline \multicolumn{7}{|l|}{ Número de consultas a um médico } \\
\hline \multicolumn{7}{|l|}{ nos últimos 12 meses } \\
\hline Nenhuma & 36,5 & 32,8 & 29,7 & 32,2 & 25,1 & 22,1 \\
\hline 1 & 15,2 & 15,1 & 13,9 & 15,4 & 12,9 & 13,5 \\
\hline 2 & 13,6 & 13,3 & 14,3 & 14,1 & 14,5 & 14,2 \\
\hline 3 ou + & 34,8 & 38,8 & 42,1 & 38,4 & 47,6 & 50,3 \\
\hline \multicolumn{7}{|l|}{ Tempo decorrido após a última visita } \\
\hline \multicolumn{7}{|l|}{ ao dentista (anos) } \\
\hline$<1$ & 16,6 & 11,2 & 7,1 & 21,3 & 15,8 & 9,7 \\
\hline $1-2$ & 11,8 & 7,7 & 5,5 & 13,4 & 9,0 & 6,4 \\
\hline 3 ou + & 65,3 & 72,2 & 76,9 & 59,0 & 68,6 & 72,7 \\
\hline Nunca & 6,3 & 8,8 & 10,5 & 6,2 & 6,6 & 11,2 \\
\hline \multicolumn{7}{|l|}{ Número de hospitalizações nos últimos 12 meses } \\
\hline Nenhuma & 88,0 & 83,7 & 82,2 & 89,5 & 84,5 & 80,4 \\
\hline 1 & 8,6 & 10,7 & 11,8 & 7,7 & 10,2 & 12,8 \\
\hline $2 \mathrm{ou}+$ & 3,4 & 5,6 & 6,0 & 2,9 & 5,4 & 6,8 \\
\hline Cobertura por plano de saúde * & 26,4 & 22,9 & 21,0 & 26,9 & 28,7 & 24,8 \\
\hline
\end{tabular}

* Percentual de respostas afirmativas;

** Alimentar-se, tomar banho e/ou ir ao banheiro. 
Distribuição percentual de indicadores da condição de saúde e uso de serviços de saúde entre mulheres, segundo a faixa etária. Pesquisa Nacional por Amostra de Domicílios (PNAD), 1998, 2003 5,6.

\begin{tabular}{|c|c|c|c|c|c|c|}
\hline \multirow[t]{3}{*}{ Indicadores } & \multirow{2}{*}{\multicolumn{3}{|c|}{$\begin{array}{c}\text { PNAD } 1998 \\
\text { Faixa etária (anos) }\end{array}$}} & \multirow{2}{*}{\multicolumn{3}{|c|}{$\begin{array}{c}\text { PNAD } 2003 \\
\text { Faixa etária (anos) }\end{array}$}} \\
\hline & & & & & & \\
\hline & $60-69$ & $70-79$ & 80 e + & $60-69$ & $70-79$ & 80 e + \\
\hline \multicolumn{7}{|l|}{ Percepção da saúde } \\
\hline Muito boa/Boa & 40,3 & 34,0 & 33,4 & 44,8 & 40,0 & 38,6 \\
\hline Regular & 45,5 & 46,7 & 43,6 & 43,4 & 44,6 & 43,2 \\
\hline Ruim/Muito ruim & 14,2 & 19,3 & 22,9 & 11,8 & 15,4 & 18,2 \\
\hline Interrupção das atividades habituais por & 12,9 & 16,2 & 20,3 & 11,2 & 14,5 & 20,0 \\
\hline \multicolumn{7}{|l|}{ problemas de saúde nas duas últimas semanas * } \\
\hline Ter estado acamado nas duas últimas semanas ** & 8,9 & 11,1 & 16,4 & 7,6 & 9,8 & 15,1 \\
\hline \multicolumn{7}{|l|}{ Grau de dificuldade para realizar atividades } \\
\hline \multicolumn{7}{|l|}{ da vida diária *夫 } \\
\hline Nenhum & 89,0 & 80,4 & 63,3 & 91,1 & 83,6 & 63,9 \\
\hline Pequeno & 7,6 & 11,4 & 16,4 & 5,6 & 9,2 & 14,7 \\
\hline Grande & 2,7 & 5,9 & 12,4 & 2,5 & 4,7 & 11,2 \\
\hline Não consegue & 0,8 & 2,3 & 7,9 & 0,8 & 2,5 & 10,2 \\
\hline \multicolumn{7}{|l|}{ Doenças crônicas auto-referidas } \\
\hline Hipertensão & 48,1 & 53,1 & 48,9 & 53,3 & 58,8 & 54,9 \\
\hline Artrite/Reumatismo & 40,0 & 47,7 & 49,2 & 29,6 & 36,0 & 39,1 \\
\hline Diabete & 10,4 & 13,4 & 11,9 & 13,3 & 15,2 & 13,2 \\
\hline \multicolumn{7}{|l|}{ Número de doenças crônicas auto-referidas } \\
\hline Nenhuma & 19,8 & 14,9 & 15,6 & 22,0 & 17,4 & 18,8 \\
\hline 1 & 23,2 & 21,2 & 21,2 & 26,4 & 25,1 & 25,9 \\
\hline 2 & 23,7 & 24,8 & 25,9 & 23,1 & 23,6 & 23,4 \\
\hline 3 ou + & 33,3 & 39,2 & 37,3 & 28,6 & 34,0 & 31,9 \\
\hline \multicolumn{7}{|l|}{ Número de consultas a um médico } \\
\hline \multicolumn{7}{|l|}{ nos últimos 12 meses } \\
\hline Nenhuma & 22,9 & 21,4 & 22,5 & 17,1 & 15,8 & 16,6 \\
\hline 1 & 13,3 & 12,5 & 12,5 & 12,0 & 10,9 & 10,6 \\
\hline 2 & 14,4 & 14,3 & 14,7 & 14,2 & 14,5 & 14,2 \\
\hline 3 ou + & 49,5 & 51,7 & 50,4 & 56,7 & 58,9 & 58,6 \\
\hline \multicolumn{7}{|l|}{ Tempo decorrido após a última visita } \\
\hline \multicolumn{7}{|l|}{ ao dentista (anos) } \\
\hline$<1$ & 15,4 & 10,4 & 5,8 & 19,9 & 14,8 & 8,5 \\
\hline $1-2$ & 10,9 & 7,7 & 5,2 & 13,1 & 8,8 & 5,9 \\
\hline 3 ou + & 69,7 & 76,2 & 79,8 & 63,2 & 70,8 & 76,8 \\
\hline Nunca & 4,1 & 5,8 & 9,2 & 3,8 & 5,5 & 8,9 \\
\hline \multicolumn{7}{|l|}{ Número de hospitalizações nos últimos 12 meses } \\
\hline Nenhuma & 88,6 & 85,7 & 80,7 & 89,6 & 86,2 & 82,5 \\
\hline 1 & 8,1 & 9,5 & 12,0 & 7,3 & 9,1 & 11,5 \\
\hline $2 \mathrm{ou}+$ & 3,4 & 4,8 & 7,3 & 3,1 & 4,7 & 6,0 \\
\hline Cobertura por plano de saúde * & 29,3 & 28,1 & 26,5 & 30,7 & 32,4 & 29,9 \\
\hline
\end{tabular}

* Percentual de respostas afirmativas;

** Alimentar-se, tomar banho e/ou ir ao banheiro. 
proporção de entrevistas respondidas por outra pessoa nessa pesquisa, porém, é muito alta, correspondendo a cerca de $1 / 3$ na faixa etária de sessenta anos ou mais 7 . Isso tem levado à apreensão quanto ao uso da informação sobre a percepção da saúde existente nos bancos de dados da PNAD. Esse questionamento baseia-se na premissa de que a auto-avaliação da saúde representa uma visão subjetiva do indivíduo, que não pode ser aferida por outra pessoa. Entretanto, um estudo recente, utilizando da PNAD 1998 e da PNAD 2003, mostrou que o uso de outro respondente não modifica a distribuição da auto-avaliação da saúde entre idosos, nem os fatores associados a ela 13. Dessa forma, no presente trabalho, optou-se por considerar toda a informação relativa à percepção da saúde, desconsiderando-se quem respondeu à entrevista.

Nossos resultados mostram o predomínio da melhor percepção da saúde da população idosa brasileira, particularmente em 2003. Essa população apresenta melhor percepção da saúde do que a população idosa norte-americana. A melhor avaliação é observada quando se considera o conjunto da população e quando se consideram as várias faixas etárias que compõem o segmento idoso 3 .

A manutenção da capacidade funcional é um dos indicadores mais importantes do envelhecimento bem-sucedido. Trata-se de um componente central da saúde do idoso, que tem sido muito importante para a formulação de conceitos como a "expectativa de vida ativa" 14 . A capacidade funcional pode ser determinada utilizando-se escalas como Atividades Instrumentais da Vida Diária e Atividades da Vida Diária, entre outros indicadores. No presente trabalho, a capacidade funcional foi avaliada, considerando-se o grau de dificuldade para realizar pelo menos uma entre três atividades da vida diária (alimentar-se, tomar banho ou ir ao banheiro), que caracterizam a dependência extrema. A PNAD inclui outros indicadores de capacidade funcional e/ou mobilidade (por exemplo, abaixar-se, ajoelhar-se ou curvar-se, caminhar por certa distância, subir ladeira ou escada e atividades que requerem maior esforço), mas existem indícios de que as prevalências de incapacidades para essas atividades estejam subestimadas na referida pesquisa 7 . Por isso, elas não foram consideradas neste trabalho.

Resultados de um estudo multicêntrico conduzido nos Estados Unidos mostraram que a total autonomia (definida como relato de nenhuma dificuldade) para alimentar-se, tomar banho ou ir ao banheiro varia entre $83 \%$ e $97 \%$ entre pessoas com 65 ou mais anos de idade 14. Os resultados do presente trabalho estão compre- endidos dentro dessa variação. A prevalência de incapacidade para alimentar-se, tomar banho ou ir ao banheiro foi a mais influenciada pela idade, entre todas as características consideradas neste trabalho, tendo aumentado seis vezes entre os homens e dez vezes entre as mulheres nas faixas etárias extremas.

Os resultados do presente trabalho mostram que a prevalência da hipertensão arterial (55\%) e do diabetes (14\%) entre idosas foi semelhante à observada entre as idosas norte-americanas em 2002-2003 (54\% e 14\%, respectivamente). Todavia, no sexo masculino, as prevalências correspondentes no Brasil ( $40 \%$ e $12 \%$, respectivamente) foram inferiores às observadas nos Estados Unidos (47\% e 19\%, respectivamente) ${ }^{3}$. A menor prevalência de hipertensão e diabetes auto-referido entre idosos, em comparação às idosas, pode ser devido a diversos fatores, tais como menor prevalência das mesmas (em razão da menor incidência e/ou menor sobrevida), menor sensibilidade do instrumento utilizado para obter a informação (ver comentário abaixo), menor probabilidade do diagnóstico devido a menor uso de serviços de saúde e/ou uma combinação desses fatores.

A prevalência de artrite/reumatismo entre idosos, observada neste trabalho $(27 \%)$ para o ano de 2003, foi inferior à observada no Canadá (40\%) 15 e nos Estados Unidos (59\%) 16, mas a razão entre os sexos foi semelhante à observada nesses países. Esse resultado pode estar refletindo a menor prevalência de artrite/reumatismo entre idosos brasileiros, mas não se pode descartar a hipótese da ausência de diagnóstico. Fala a favor da última hipótese a redução da prevalência de artrite/reumatismo observada entre 1998 e 2003, conforme será comentado a seguir.

As tendências da morbidade auto-referida, baseadas nas informações produzidas pela PNAD em 1998 e 2003, devem ser encaradas com alguma reserva porque a forma como a informação foi obtida diferiu nos dois períodos. Em 1998, a pergunta adotada foi “Tem ... [tal] doença?" Em 2003, essa pergunta foi substituída por "Algum médico ou profissional de saúde já disse que você tem ... [tal] doença?". A modificação foi pertinente porque a última pergunta é universalmente usada e tem validade conhecida para algumas doenças; no entanto isso pode ter resultado em estimativas de prevalências mais influenciadas pelo acesso aos serviços de saúde em 2003 do que em 1998.

Trabalhos recentes realizados nos Estado Unidos, com base na pergunta adotada pela PNAD em 2003, mostraram boa sensibilidade e, sobretudo, especificidade para a hipertensão (71\% e 92\%, respectivamente) 17 e diabetes auto- 
referidos (73\% e 99\%, respectivamente) 18 . Valores muito semelhantes para hipertensão arterial (72\% e 86\%, respectivamente) foram observados em um estudo de base populacional brasileiro, conduzido na cidade de Bambuí, Minas Gerais, Brasil. Nele se observou um aumento da sensibilidade com a idade, alcançando valor mais alto entre idosos (81\%) e entre as mulheres em comparação aos homens (79\% e $61 \%$, respectivamente) 19. Como comentado anteriormente, a influência do sexo na validade do instrumento utilizado pode explicar - pelo menos em parte - diferenças na prevalência da morbidade autoreferida para algumas doenças.

Com relação ao uso de serviços de saúde, observou-se um aumento significativo das consultas médicas entre 1998 e 2003. O aumento das consultas médicas parece ter sido em função da melhoria do acesso. Uma explicação alternativa seria um aumento das necessidades em saúde entre 1998 e 2003. A primeira hipótese é reforçada por dois outros resultados: (1) os indicadores das condições de saúde apontam para melhor performance em 2003, em comparação a 1998; (2) o número de hospitalizações não se modificou nesse período. É importante salientar que não houve mudança significativa na cobertura por plano de saúde da população idosa brasileira, entre 1998 e 2003, permanecendo a sua maioria (cerca de 70\%) na dependência do SUS.

A população idosa é o segmento da população que mais usa os serviços de saúde, por causa do aumento das doenças crônicas e suas conseqüências, com importantes repercussões para o sistema de saúde. No Brasil, a razão entre o custo proporcional das hospitalizações no âmbito do SUS e o tamanho proporcional da população idosa é igual a 2,6, observando-se o seu aumento gradual com a idade $(2,2 ; 3,1$ e 3,7 nas faixas etárias de 60-69, 70-79 e 80 e + anos de idade) 20. Os resultados do presente trabalho são coerentes com tais observações. O número de hospitalizações aumentou gradualmente com a idade, em ambos os sexos e nos dois períodos estudados. Padrão semelhante foi observado para o número de consultas médicas entre os homens, que aumentou com a idade. Entretanto, esse padrão não foi observado entre as mulheres. Como esperado, as idosas de todas as faixas etárias consultaram mais os médicos do que os idosos, não se observando modificação importante com a idade.

Considerando-se o objetivo deste trabalho, que era examinar as tendências das condições de saúde e do uso de serviços de saúde entre idosos brasileiros, a PNAD apresenta algumas limitações e muitas vantagens. Entre as limitações, destaca-se a insuficiência da informação sobre capacidade funcional. Essa informação é limitada a somente três das diversas atividades da vida diária, não incluindo, por exemplo, a capacidade para vestir-se, para transferir-se da cama para uma cadeira ou caminhar pela casa, entre outras ${ }^{3}$. Tradicionalmente, as perguntas relativas às atividades da vida diária são individuais, ou seja, obtém-se a informação sobre o grau de dificuldade para realizar cada atividade. Na PNAD, a informação sobre as três atividades é obtida por meio de uma única pergunta. Isso dificulta a comparação dos resultados brasileiros com os de outras populações. A segunda limitação refere-se à possível subestimativa das prevalências referentes aos demais indicadores de função física e/ou mobilidade, pela forma com a qual a pergunta é feita no questionário da PNAD 7. A terceira limitação refere-se à ausência de informações sobre estilos de vida (tais como atividades físicas e tabagismo), assim como uso de serviços preventivos de saúde. Na PNAD 2003, foram incluídas perguntas sobre mamografia e exame de Papanicolau, mas não existem informações sobre pesquisa de sangue oculto nas fezes, freqüência de medidas de pressão arterial e dosagem de colesterol, que são importantes para a prevenção secundária, e objeto de monitoramento em alguns países 21. Por outro lado, a PNAD apresenta vantagens que são únicas. Entre as vantagens, podem ser citadas: abrangência nacional da pesquisa; sua periodicidade (1998 e 2003); qualidade da informação; delineamento amostral; inclusão de uma gama de assuntos que permitem investigar as condições de saúde da população brasileira e seus determinantes sob diversos prismas, entre outras. O tamanho da amostra é especialmente relevante em estudos da população idosa. Essa população representa o segmento que mais cresce da população brasileira, mas representa ainda menos de $10 \%$ da população total do país 22,23 . Além disso, trata-se de um grupo heterogêneo, com amplitude de idade que pode chegar a quarenta anos. Essa heterogeneidade exige que a amostra seja grande o suficiente para permitir a estratificação pela idade. Não existem inquéritos nacionais com essa característica. Os bons inquéritos brasileiros excluíram a população idosa ou trataram esse grupo como um estrato homogêneo. Isso não acontece na PNAD, que inclui cerca de 30 mil idosos na amostra. Por isso, o suplemento de saúde da PNAD é o primeiro inquérito com abrangência nacional que permite inferência para a população idosa brasileira (exceto interior da Região Norte) e as diversas faixas etárias que compõem esse segmento populacional.

Os resultados do presente trabalho indicam que as informações sobre saúde - e aspectos re- 
lacionados-existentes no suplementos de saúde da PNAD podem ser úteis para o monitoramento e/ou vigilância das condições de saúde da população idosa brasileira. A análise dos dados dessa pesquisa mostrou consistência interna e coerência com os conhecimentos existentes sobre as condições de saúde da população idosa. A manutenção desse suplemento e a garantia da sua periodicidade são muito importantes para que as tendências das condições de saúde da população idosa brasileira possam ser permanentemente avaliadas. A base de dados da PNAD 1998 e 2003 permitiu identificar melhora nas condições de saúde auto-referidas da população idosa brasileira, assim como um aumento no número de consultas médicas. Esse aumento parece terse devido à melhora de acesso, possivelmente no âmbito do SUS.

\section{Resumo}

Este trabalho teve por objetivo verificar as tendências das condições de saúde e do uso de serviços de saúde entre idosos brasileiros, utilizando dados da Pesquisa Nacional por Amostras de Domicílios (PNAD). Foram incluídos no estudo 28.943 e 35.042 participantes das PNAD 1998 e 2003 com idade $\geq 60$ anos, respectivamente. Os resultados mostraram que houve melhora nas condições de saúde dos idosos nesse período, considerando-se indicadores como percepção da saúde, ter estado recentemente acamado, capacidade para realizar atividades da vida diária e número de doenças crônicas. Os resultados também monstraram que houve aumento do número de consultas médicas e odontológicas entre 1998 e 2003. Tais tendências foram consistentemente observadas em ambos os sexos. Os autores chamam a atenção para a importância do suplemento de saúde da PNAD como fonte de informação para o monitoramento elou vigilância das condições de saúde da população idosa brasileira.

Idoso; Serviços de Saúde; Pesquisa sobre Serviços de Saúde; Nível de Saúde

\section{Colaboradores}

M. F. Lima-Costa delineou o estudo e redigiu o manuscrito. A. I. Loyola Filho e D. L. Matos prepararam os bancos de dados, realizaram a análise dos dados e participaram da revisão crítica do manuscrito.

\section{Agradecimentos}

Este trabalho foi desenvolvido como parte das atividades do Núcleo de Estudos sobre Saúde Pública e Envelhecimento, Fundação Oswaldo Cruz/Universidade Federal de Minas Gerais, como Centro Colaborador em Saúde do Idoso, Secretaria de Vigilância à Saúde, Ministério da Saúde. A pesquisa contou com recursos do Ministério da Saúde. M. F. Lima-Costa é pesquisador bolsista do Conselho Nacional de Desenvolvimento Científico e Tecnológico (CNPq). 


\section{Referências}

1. Last L. A dictionary of epidemiology. $4^{\text {th }}$ Ed. Oxford: Oxford University Press; 2001.

2. Government of Canada. National advisory council on aging. Report card seniors in Canada. Québec: Government of Canada; 2001.

3. National Center for Health Statistics, Centers for Disease Control and Prevention. Trends in health and aging. http://www.cdc.gov/nchs/agingact. htm (acessado em 13/Out/2005).

4. Secretaria de Vigilância à Saúde, Ministério da Saúde. Sistemas de informações. http://portal.saude. gov.br/portal/svs/area.cfm?id_area $=460$ (acessado em 20/Nov/2005).

5. Instituto Brasileiro de Geografia e Estatística. Pesquisa nacional por amostras de domicílios. Rio de Janeiro: Instituto Brasileiro de Geografia e Estatística; 1998.

6. Instituto Brasileiro de Geografia e Estatística. Pesquisa nacional por amostras de domicílios. Rio de Janeiro: Instituto Brasileiro de Geografia e Estatística; 2003.

7. Lima-Costa MF, Barreto S, Giatti L. Condições de saúde, capacidade funcional, uso de serviços de saúde e gastos com medicamentos da população idosa brasileira: um estudo descritivo baseado na Pesquisa Nacional por Amostra de Domicílios. Cad Saúde Pública 2003; 19:735-43.

8. Hosmer DW, Lemeshow S. Applied logistic regression. New York: John Wiley \& Sons; 1989.

9. Idler EL, Benyamini Y. Self-rated health and mortality: a review of twenty-seven community studies. J Health Soc Behav 1997; 38:21-37.

10. Jylhä M, Guralnik JM, Ferrucci L, Jokela J, Heikkinen E. Is self-rated health comparable across cultures and genders? J Gerontol B Psychol Sci Soc Sci 1998; 53:S144-52.

11. Idler EL, Kasl SV. Self-ratings of health: do they also predict change in functional ability? J Gerontol B Psychol Sci Soc Sci 1995; 50:S344-53.

12. Grundy E, Sloggett A. Health inequalities in the older population: the role of personal capital, social resources and socio-economic circumstances. Soc Sci Med 2003; 56:935-47.
13. Lima-Costa MF, Peixoto SV, Matos DL, Firmo JOA, Uchôa E. A influência de respondente substituto na percepção da saúde de idosos: um estudo baseado na Pesquisa Nacional por Amostra de Domicílios $(1998,2003)$ e na coorte de Bambuí, Minas Gerais, Brasil. Cad Saúde Pública 2007; 23:1893-902.

14. Cornoni-Huntley J, Brock DB, Ostfeld AM, Taylor JO, Wallace RB. Established populations for epidemiologic studies of the elderly. Washington DC: US Department of Health and Human Services, National Institutes of Health; 1986. (NIH Publication 86-2443).

15. Bradley EM, Wang PP. Arthritis and the aging population; projections of arthritis prevalence in Canada 1991 to 2003. Rheumatology 1998; 25:138-44.

16. Centers for Disease Control and Prevention. Prevalence of self-reported arthritis of chronic joint symptoms among adults - United States 2001. MMWR Morb Mortal Wkly Rep 2002; 51:948-50.

17. Vargas CM, Burt VL, Gillum RF, Pamuk ER. Validity of self-reported hypertension in the National Health and Nutrition Examination Survey III, 1988-1991. Prev Med 1997; 26:678-85.

18. Martin LM, Leffe N, Calonge N, Garrett C, Nelson DE. Validation of self-reported chronic conditions and health services in a managed care population. Am J Prev Med 2000; 18:215-8.

19. Lima-Costa MF, Peixoto SV, Firmo JOA. Validade da hipertensão auto-referida e seus determinantes (projeto Bambuí). Rev Saúde Pública 2004; 38:63742.

20. Peixoto SV, Giatti L, Afradique ME, Lima-Costa MF. Custo das internações hospitalares entre idosos brasileiros no âmbito do Sistema Único de Saúde. Epidemiol Serv Saúde 2004; 13:239-46.

21. Centers for Disease Control and Prevention. Healthy people 2010. http://healthypeople.gov (acessado em 20/Nov/2005).

22. Instituto Brasileiro de Geografia e Estatística. Censo demográfico. Rio de Janeiro: Instituto Brasileiro de Geografia e Estatística; 1992.

23. Instituto Brasileiro de Geografia e Estatística. Censo demográfico. Rio de Janeiro: Instituto Brasileiro de Geografia e Estatística; 2002.

Recebido em 24/Abr/2006

Versão final reapresentada em 07/Ago/2006

Aprovado em 31/Ago/2006 